\title{
Prospective study of hallucinations and delusions in Parkinson's disease
}

\author{
S Holroyd, L Currie, G F Wooten
}

\begin{abstract}
Objective-This study was undertaken to determine the prevalence of hallucinations and delusions in Parkinson's disease, to describe such symptoms phenomenologically, and possibly to determine factors associated with their development. In addition, the role of the visual system in relation to visual hallucinations was examined.

Method-102 consecutive patients diagnosed with strictly defined Parkinson's disease were examined for the presence of hallucinations and delusions and assessed for visual acuity, cognition, depression using the geriatric depression scale, disease severity as measured by the UPDRS, and other clinical variables.
\end{abstract}

Results-Of 102 consecutive patients, $29.4 \%(n=30)$ had hallucinations or delusions, four $(3.9 \%)$ were determined to be psychotic due solely to delirium and were excluded from further analysis. Of the 98 remaining patients, $26.5 \% \quad(n=26)$ had visual hallucinations. Among these, one patient also had delusions, two had auditory hallucinations, and one had gustatory hallucinations. Visual hallucinations were significantly associated with worse visual acuity, lower cognitive score, higher depression score, and worse disease severity. Hallucinations were not associated with history of psychiatric disease, dose or duration of levodopa or other antiparkinsonian medication treatment, or duration of illness.

Conclusions-Visual hallucinations are common symptoms in Parkinson's disease and are most likely of multifactorial origin. Although higher doses of levodopa are known to be related clinically to hallucinations in individual patients, the results suggest that several underlying characteristics of patients with Parkinson's disease (disease severity, dementia, depression, worse visual acuity) may be more important determinants of which patients experience hallucinations. The data also provide preliminary evidence that abnormality of the visual system may be related to visual hallucinations in Parkinson's disease, as has been found in other disorders with visual hallucinations. (F Neurol Neurosurg Psychiatry 2001;70:734-738)

Keywords: visual hallucinations; Parkinson's disease; delusions
Hallucinations and delusions occur in patients with Parkinson's disease with reported prevalences ranging from $6 \%$ to $40 \% .^{1}$ Such symptoms are associated with major behavioural and functional problems, ${ }^{2}$ are a risk factor for nursing home placement ${ }^{3}$ and are associated with higher mortality. ${ }^{4}$ For patients and their families, hallucinations and delusions can decrease the quality of life and increase the burden of the illness. ${ }^{56}$

Because of the serious consequences of these symptoms, additional research in this area is needed. Several methodological problems plague studies of hallucinations and delusions in Parkinson's disease. For one, multiple illnesses can result in parkinsonism, ${ }^{7}$ yet some studies have not specified the precise diagnoses of patients studied, or have lumped patients with different neurological diagnoses together. In addition, the aetiology and phenomenology of the hallucinations and delusions are often not described, making it unclear as to the cause and quality of these symptoms. Patients are often labelled with terms such as "dopaminergic psychosis" or "Parkinson's disease psychosis" despite the knowledge that patients with Parkinson's disease may have hallucinations and delusions from causes including changes in the brain from the illness itself, delirium, or other psychiatric disorders. ${ }^{5}$

A recent comprehensive review concluded that there are six psychotic disorders in Parkinson's disease: (1) hallucinations with preserved insight; (2) medication induced psychotic disorders in clear consciousness but without insight; (3) delirium, (4) schizophrenia-like psychotic disorders which occur in clear consciousness and without levodopa treatment; (5) schizophrenia with subsequent development of Parkinson's disease; and (6) other psychotic disorders including major depression or mania." "The continuum hypothesis" has been proposed suggesting that medication induced psychiatric symptoms in Parkinson's disease begin with sleep disruption, then vivid dreams, and progress to hallucinatory and delusional experiences ending in delirium. ${ }^{8} 9$

Despite the large literature linking hallucinations in Parkinson's disease to its treatment with dopaminergic and anticholinergic medication, only some patients experience hallucinations, suggesting there may be predisposing factors. A community based study of 245 patients with Parkinson's disease disclosed psychosis to be associated with advanced age, stage of Parkinson's disease, depression, and cognitive impairment. Interestingly, antiparkinson drug therapy was not found to be associated with psychosis. ${ }^{10}$ A population based 
study of 775 patients with Parkinson's disease, however, did not find a relation between hallucinations and stage or severity of Parkinson's disease ${ }^{11}$ Other studies have showed hallucinations to be associated with dementia, and history of depression or sleep disorder. ${ }^{12}{ }^{13}$ Other studies have also found an association between hallucinations and lower cognitive scores. ${ }^{1415}$

Notably, in all studies, the hallucinations have been found to be almost entirely visual in nature. ${ }^{1}{ }^{101216}$ Visual hallucinations in other disorders, including Alzheimer's disease, have been associated with dysfunction in the visual system including decreased visual acuity and visual agnosia. ${ }^{17-19} \mathrm{~A}$ study examining visual discrimination tasks showed that patients with Parkinson's disease with visual hallucinations had worse performance than patients without visual hallucinations..$^{20}$ To our knowledge, this relation between visual dysfunction and visual hallucinations has not been further explored in Parkinson's disease.

The aim of this study was to prospectively examine patients with strictly defined Parkinson's disease to establish a prevalence of hallucinations and delusions, to describe such symptoms phenomenologically, and to examine possible associations with demographic and clinical variables. In addition, given the relation between visual hallucinations and visual acuity in Alzheimer's disease, a hypothesis of the study was that visual hallucinations in Parkinson's disease would be associated with decreased visual acuity.

\section{Methods}

One hundred and two consecutive patients seen at the University of Virginia movement disorder clinic diagnosed with Parkinson's disease were examined in this study. Diagnosis was made by movement disorder subspecialists using internationally accepted criteria. ${ }^{21-23} \mathrm{At}$ least two of the following three criteria had to be present: rest tremor, cogwheel rigidity, and bradykinesia. Asymmetry of these features at the time of diagnosis and at onset had to be present. Exclusion criteria included the presence of atypical features (for example, supranuclear vertical gaze palsy, prominent, early orthostatic hypotension, apraxia, parietal lobe sensory dysfunction), presence of a preexisting possible cause (for example, history of multiple strokes, history of encephalitis), definite absence of response to levodopa (dosage up to $600 \mathrm{mg} /$ day), or a clearly non-progressive course over at least 3 years. All patients meeting these criteria consented to participate in the study.

Patients were evaluated for the following demographic information: age, sex, education, race, living situation, treatment with antipsychotic drugs, and personal history of psychiatric disorder. Patients were screened for delusions and hallucinations, information being taken both from the patient and the caregiver or informant if available. Patients meeting DSM-IV criteria $^{24}$ for delirium were excluded from further analysis of the study, as a major goal was to examine psychosis associated with
Parkinson's disease and not due to other causes. Patients were also assessed for presence of schizophrenia using DSM-IV criteria. $^{24}$ Visual hallucinations were defined (as in studies of hallucinations in Alzheimer's disease) if (1) patients reported seeing a perception in the absence of an external stimulus or (2) if the caregiver, by history, or the examiner, saw the patients interact by verbalisation or behaviour with a non-existent person or object. ${ }^{17}$ Patients had to have experienced visual hallucinations at least once in the past week to be included. A description of the visual hallucination was also obtained. Hallucinations of other modalities including auditory, olfactory, tactile, and gustatory hallucinations and delusions were similarly assessed. Visual acuity was measured using the Snellen method. Patients wore their glasses for testing, and a measure of the acuity of the "best eye" was obtained.

Patients reporting hallucinations or delusions were then asked what caused these experiences, in an effort to assess the patient's insight. Informants were also asked about the level of the patient's insight. A cognitive testthe telephone interview for cognitive status (TICS) $)^{25}$-was given. The TICS was used as it does not depend on motor or visual capability, both which were thought to be possibly compromised in patients with Parkinson's disease. The TICS correlates very highly with the Folstein mini mental state examination ${ }^{26}$ with a Pearson correlation of 0.94 . The TICS is scored from 0 to 41 , normal subjects scoring from 31 to 41 . The TICS has a sensitivity of $94 \%$ and specificity of $100 \%$ for classifying persons with dementia. The TICS was administered in person, as has been done in prior studies. ${ }^{17}{ }^{19}$ Patients were also administered the geriatric depression scale (GCS), ${ }^{27}$ which has been validated in elderly and medically ill patients including those with mild to moderate dementia. ${ }^{28-30}$ The GDS consists of 30 yes-no questions where higher scores indicate more depressive symptoms. Scores of 11 or greater indicate possible depression with an $84 \%$ sensitivity and a $95 \%$ specificity rate. ${ }^{27}$

Each patient was interviewed about disease onset and medication history including current dopaminergic and other antiparkinsonian medications and dosage. These included levododopa, selegilene, anticholinergic drugs, amantadine, and dopamine agonists. In addition, patients were evaluated using the unified Parkinson's disease rating scale (UPDRS). ${ }^{31}$ The UPDRS is composed of three subscales: mentation, behaviour, and mood (four items); activities of daily living (ADL; 13 items); and motor (27 items). Scores for each item may range from 0 (normal or absent) to 4 (severe) and yield a composite UPDRS score that may range from 0 to 176 , where higher scores are indicative of increased severity of disease. Patients were fully informed of the nature and intent of the study. After complete description of the study to the patients, written informed consent was obtained.

Data were examined using the SPSS/PC+ statistical program. First a prevalence of 
psychotic symptoms due to all causes (including delirium) was determined. Next, patients whose psychosis was thought to be secondary to delirium only and not Parkinson's disease, as determined by DSM IV criteria, were eliminated from further analysis. Next, multvariate analysis of variance (MANOVA) (Wilks'Lambda) was performed to determine if the presence of hallucinations influenced any of the dependent variables (TICS, visual acuity, GDS, UPDRS, total levodopa dose, duration of levodopa treatment, other antiparkinsonian medication use and dosage, number of medications). Based on the outcome of the MANOVA, each dependent variable was then examined using student's $t$ test or Fisher's exact test as appropriate. A p value $<0.05$ was used to test for significance and all $\mathrm{p}$ values are two tailed.

\section{Results}

One hundred and two patients were examined in the study with $29.4 \%(n=30)$ having hallucinations or delusions. Of these, $3.9 \% \quad(n=4)$ were thought to have psychosis secondary solely to delirium. Demographics of the remaining 98 patients are shown in table 1 . There were no differences between hallucinators and non-hallucinators on any demographic variable. Current use of levodopa or other antiparkinson medication is also shown in table 1. Examination of levodopa therapy disclosed a mean total daily levodopa dose of 607.7 (SD 505.5) mg. Duration of treatment with levodopa disclosed a mean of 7.9 (SD 4.6) years.

Of the 98 patients studied, $26.5 \% \quad(n=26)$ had visual hallucinations. Of these, several had other additional symptoms. One patient had delusions $(1.0 \%)$, two patients had auditory hallucinations $(2.0 \%)$, and one patient had gustatory hallucinations $(1.0 \%)$. Further analysis comparing patients with solely visual hallucinations with those with visual hallucinations plus another hallucination or delusion was not possible due to the few patients with more than one symptom. Duration of visual hallucinations ranged from one to 48 months

Table 1 Demographics and clinical characteristics of sample $(n=98)$

\begin{tabular}{|c|c|c|c|}
\hline & Mean (SD) & $\%$ & $n$ \\
\hline Age (y) & $67.5(9.1)$ & & \\
\hline \multicolumn{4}{|l|}{ Sex: } \\
\hline Male & & 60.2 & 59 \\
\hline Female & & 39.8 & 39 \\
\hline \multicolumn{4}{|l|}{ Race: } \\
\hline White & & 96.9 & 95 \\
\hline African-American & & 2 & 2 \\
\hline Other & & 1 & 1 \\
\hline Level of education (y) & $14.7(3.8)$ & & \\
\hline \multicolumn{4}{|l|}{ Living situation: } \\
\hline Alone & & 15.3 & 15 \\
\hline Spouse/caregiver & & 81.6 & 80 \\
\hline Nursing home & & 3.1 & 3 \\
\hline Age of onset (PD) & $59.4(10.3)$ & & \\
\hline Duration of disease $(y)$ & $9.7(5.3)$ & & \\
\hline \multicolumn{4}{|l|}{ Current use of: } \\
\hline Levodopa & & 83.7 & 82 \\
\hline Anticholinergic drugs & & 15.3 & 15 \\
\hline Amantadine & & 9.2 & 9 \\
\hline Dopamine agonists & & 27.6 & 27 \\
\hline Selegilene & & 52.0 & 51 \\
\hline
\end{tabular}

Table 2 Characteristics of visual hallucinations in 26 patients with Parkinson's disease

\begin{tabular}{|c|c|c|}
\hline Characteristic & Patient No & $\%$ \\
\hline \multicolumn{3}{|l|}{ Frequency: } \\
\hline Daily & 6 & 23.1 \\
\hline 2-4 times/week & 6 & 23.1 \\
\hline Once a week & 9 & 34.6 \\
\hline Don’t know & 5 & 19.2 \\
\hline \multicolumn{3}{|l|}{ Size: } \\
\hline Normal & 12 & 46.2 \\
\hline Miniaturised & 9 & 34.6 \\
\hline Don't know & 5 & 19.2 \\
\hline \multicolumn{3}{|l|}{ Transparency: } \\
\hline Solid & 25 & 96.2 \\
\hline Transparent & 1 & 3.8 \\
\hline \multicolumn{3}{|l|}{ Movement: } \\
\hline Moves & 7 & 26.9 \\
\hline Still & 17 & 65.4 \\
\hline Varies & 2 & 7.7 \\
\hline \multicolumn{3}{|l|}{ Content } \\
\hline People & 15 & 57.7 \\
\hline Animals & 7 & 26.9 \\
\hline Objects & 8 & 30.8 \\
\hline Can't describe & 2 & 7.7 \\
\hline Multiple content & 19 & 73.1 \\
\hline Single content & 5 & 19.2 \\
\hline Can’t describe & 2 & 7.7 \\
\hline \multicolumn{3}{|c|}{ Familiarity of content: } \\
\hline Familiar & 6 & 23 \\
\hline Unfamiliar & 18 & 69.2 \\
\hline Unknown & 2 & 7.7 \\
\hline \multicolumn{3}{|l|}{ Duration: } \\
\hline Minutes & 2 & 7.7 \\
\hline Hours & 8 & 30.8 \\
\hline Days & 2 & 7.7 \\
\hline Varies & 10 & 38.5 \\
\hline
\end{tabular}

with a mean duration of 11.7 (SD 14.1) months.

Further description of the visual hallucinations are shown in table 2. The content of the hallucinations included "miniaturised armies at war", "Mafia men", "running black horses", "large buildings", "yellow ribbons fluttering in the breeze", "lambs and tigers", and one reported seeing himself. There seemed to be a continuum among those patients with insight to those without insight that the hallucinations were not real. Some patients claimed to have current insight into their hallucinations but admitted, or care givers reported at times becoming distressed or behaving as if the hallucinations were real. Of the 26 hallucinators, four had no insight that the hallucinations were not real whereas 12 were thought to have full insight and the remaining 10 patients had partial or variable insight. Analysis of cognitive score between these groups showed no difference between those with full insight, partial insight, or no insight (one way ANOVA $F=0.514, \mathrm{p}=0.605)$.

A MANOVA (Wilks' Lambda) was performed to determine if the presence of hallucinations was associated with any of the dependent variables, disclosing a $\mathrm{p}$ value of 0.005 . Based on this result, each dependent variable was examined by either student's $t$ test or Fisher's exact test as appropriate, comparing hallucinators to non-hallucinators. A summary of these comparisons is shown in table 3.

Hallucinations were associated with a lower cognitive score, worse vision as measured by lower visual acuity in the patients "best eye", a higher score on the GDS, and a higher score on the disease severity scale (UPDRS). Further examination of subscale measures within the UPDRS disclosed a significant association with 
Table 3 Comparison of hallucinators v non-hallucinators

\begin{tabular}{|c|c|c|c|c|c|}
\hline & \multicolumn{2}{|c|}{ Visual hallucinations $(n=26)$} & \multicolumn{2}{|c|}{ No hallucinations $(n=72)$} & \multirow[b]{2}{*}{ Statistical analysis } \\
\hline & Mean $(S D)$ & $n(\%)$ & Mean (SD) & $n(\%)$ & \\
\hline TICS & $29.9(5.8)$ & & $33.0(3.5)$ & & $t=-3.22, \mathrm{df}=95, \mathrm{p}=0.002$ \\
\hline Visual acuity best eye 20 / & $20 / 44.6(36.6)$ & & $20 / 32.8(12.1)$ & & $t=2.42, \mathrm{df}=95, \mathrm{p}=0.018$ \\
\hline GDS & $7.9(6.5)$ & & $5.0(4.5)$ & & $t=2.50, \mathrm{df}=95, \mathrm{p}=0.014$ \\
\hline UPDRS & $47.8(21.4)$ & & $38.5(16.5)$ & & $t=2.22, \mathrm{df}=91, \mathrm{p}=0.03$ \\
\hline Total levodopa dose (mg/d) & $648.1(533.9)$ & & $593.1(497.9)$ & & $t=0.47, \mathrm{df}=96, \mathrm{p}=0.63$ \\
\hline Years of levodopa treatment & $9.2(4.3)$ & & $7.4(4.7)$ & & $t=1.63, \mathrm{df}=79, \mathrm{p}=0.11$ \\
\hline No of medications & $4.6(2.0)$ & & $4.0(2.1)$ & & $t=1.20, \mathrm{df}=91, \mathrm{p}=0.23$ \\
\hline \multicolumn{6}{|l|}{ Personal psychiatric history: } \\
\hline Yes & & $9(34.6)$ & & $19(26.4)$ & Fisher's exact test \\
\hline No & & $17(65.4)$ & & $53(73.6)$ & $\mathrm{p}=0.30$ \\
\hline \multicolumn{6}{|l|}{ Levodopa use: } \\
\hline Yes & & $22(84.6)$ & & $60(83.3)$ & Fisher's exact test \\
\hline No & & $4(15.4)$ & & $12(16.7)$ & $\mathrm{p}=1.0$ \\
\hline \multicolumn{6}{|l|}{ Anticholinergic use: } \\
\hline Yes & & $5(19.2)$ & & $10(14.1)$ & Fisher's exact test \\
\hline No & & $21(80.8)$ & & $61(85.9)$ & $\mathrm{p}=0.54$ \\
\hline \multicolumn{6}{|l|}{ Amantadine use: } \\
\hline Yes & & $4(15.4)$ & & $5(7.0)$ & Fisher's exact test \\
\hline No & & $22(84.6)$ & & $66(93.0)$ & $\mathrm{p}=0.24$ \\
\hline \multicolumn{6}{|l|}{ Dopamine agonist use: } \\
\hline Yes & & $10(38.5)$ & & $17(24.3)$ & Fisher's exact test \\
\hline No & & $16(61.5)$ & & $53(75.7)$ & $\mathrm{p}=0.21$ \\
\hline \multicolumn{6}{|l|}{ Selegilene use: } \\
\hline Yes & & $16(61.5)$ & & $35(49.3)$ & Fisher's exact test \\
\hline No & & $10(38.5)$ & & $36(50.7)$ & $\mathrm{p}=0.36$ \\
\hline
\end{tabular}

the ADL subscale only (hallucinators 15.5 (SD 8.5) v non-hallucinators 11.4 (SD 5.6), $t=2.70$, $\mathrm{df}=93, \mathrm{p}=0.008)$. Interestingly there was no association with current use or dose of dopaminergic or other use of antiparkinson medication. Duration of levodopa treatment was also not found to be associated. Four of the hallucinators in fact were not taking levodopa at the time of the examination.

\section{Discussion}

In this sample of patients with strictly defined Parkinson's disease, prevalence of hallucinations and delusions was almost $30 \%$. This is consistent with the prevalence of 33\% hallucinations in a population based study, ${ }^{11}$ despite the possibility that our sample was skewed due to the tertiary care referral patterns to our clinic. Ours is the first study to attempt to differentiate categories of hallucinations and delusions - for example, delirium - and establish the prevalence of each. Using the categories of Peyser et at however, proved difficult. Some patients did not have fully preserved insight (Peyser category 1) or the psychosis was not clearly medication induced (Peyser category 2). Further, because the insight of some patients seemed to fluctuate, using insight as a defining criteria proved problematic. We would propose the following categories, based on our data and the work of others, showing the following prevalences:

(1) Hallucinations/delusions in clear consciousness $25.6 \%(n=26)$

(2) Delirium $3.9 \%(n=4)$

(3) Schizophrenic-like psychotic disorders in clear consciousness $0 \%$

(4) Schizophrenia with subsequent development of Parkinson's disease $0 \%$

(5) Other $0 \%$.

It is recognised that larger samples could well include psychotic disorders of categories 3, 4, or 5. However, it seems that the most common types of psychosis in Parkinson's disease are hallucinations/delusions in clear consciousness and delirium. Given the cross sectional design of our study, evidence for the "continuum hypothesis"7 8 could not be directly examined. Longitudinal studies are needed to determine whether patients move between symptoms of sleep disturbance to delirium. Interestingly, the four delirious patients in our study did not have a history of psychosis before the delirium psychosis, possibly arguing against this hypothesis. In addition, two studies have demonstrated that hallucinations and illusions in Parkinson's disease are not transitory or fluctuating phenomena but their presence was stable over an 18 month-5 year period, ${ }^{42}$ arguing against the continuum hypothesis.

The phenomenology of the hallucinations are consistent with and similar to visual hallucinations associated with macular degeneration but not patients with Alzheimer's disease. ${ }^{17} 19$ Patients with Parkinson's disease were more able to describe their hallucinations in detail than patients with Alzheimer's disease ${ }^{17}$ and had more insight into their hallucinations than patients with Alzheimer's disease. Patients with Alzheimer's disease also had much higher prevalences of other psychotic symptoms such as auditory hallucinations and delusions. ${ }^{17}$ The similarity of visual hallucinations in Parkinson's disease to those in ophthalmological disorders is interesting, as it may suggest similar pathophysiological mechanisms.

Our study supports the findings of other studies that psychotic symptoms in Parkinson's disease are associated with decreased cognition. ${ }^{14}{ }^{15}$ Interestingly, decreased cognition has also been found to be significantly associated with visual hallucinations in other disorders including macular degeneration and Alzheimer's disease. ${ }^{17} 19$ 
Our study supports an association of hallucinations with disease severity as shown by higher UPDRS scores, in particular lower ADL scores. ${ }^{10}$ A study not finding this association did not use the UPDRS to assess disease severity. ${ }^{11}$

History of psychiatric disorder was not associated with hallucinations in our sample. This is by contrast with studies that have found depression to be associated with hallucinations. ${ }^{10}{ }^{12}$ Although we found an association between higher GDS score and hallucinators, the relevance of this finding is unclear. Most hallucinators did not meet criteria for depression on the GDS. Further study may help clarify this finding.

Interestingly current levodopa use, total levodopa dose, duration of levodopa use, use of other antiparkinson medication, and number of medications were not associated with hallucinations, as found in one previous study. ${ }^{10}$ This suggests that visual hallucinations in Parkinson's disease are not simply a medication effect, but a symptom related to the disease itself, although one that may be worsened by medication. This is supported by a study finding that high dose intravenous levodopa did not precipitate hallucinations in patients with Parkinson's disease with daily hallucinations suggesting that hallucinations are not simply related to high concentrations of levodopa or sudden changes in plasma concentrations. ${ }^{33}$

The relation between decreased visual acuity and visual hallucinations in Parkinson's disease is interesting as this finding has also been found in patients with macular degeneration and in patients with Alzheimer's disease. ${ }^{17}{ }^{19}$ Although Parkinson's disease is often characterised as a pure motor system disease, sensory abnormalities have been reported including abnormal visual evoked potentials. ${ }^{34}$ It has been noted that dopaminergic circuits of the retina are involved in Parkinson's disease and that primary visual dysfunction may even contribute to visual cognitive deficits. ${ }^{35}$ Further study of the visual system in Parkinson's disease and its relation to visual hallucinations is therefore warranted.

In summary, our results suggest that psychosis in Parkinson's disease is not simply a medication effect, but is associated with the disease process itself. Our study also shows an association between visual hallucinations and the visual system, which deserves further examination. Continued research is needed to further elucidate these findings and determine the causes of hallucinations in Parkinson's disease, which may in turn lead to more successful treatments. This study was funded in part by a grant from the National
Institute of Mental Health (K07-MH01199-01A1), the American Parkinson's Disease Association and National Institute of Health P50-NS39788.

Cummings JL. Behavioral complications of drug treatment of Parkinson's disease. 7 Am Geriatr Soc 1991;39:708-16. of Parkinson's disease. F Am Geriatr Soc 1991;39:708-16. Naimark D, Jackson E, Rockwell E, et al. Psychotic
symptoms in Parkinson's disease patients with dementia. $f$ symptoms in Parkinson's disease

3 Goetz C, Stebbins G. Risk factors for nursing home placement in advanced Parkinson's disease. Neurology 1993;43:2227-9.
4 Goetz C, Stebbins G. Mortality and hallucinations in nursing home patients with advanced Parkinson's disease. Neuing home patients with
rology $1995 ; 45: 669-71$

5 Peyser CE, Naimark D, Zuniga R, et al. Psychoses in Parkinson's disease. Semin Clin Neuropsychiatry 1998;3:11

6 Melamed E, Friedberg G, Zoldan J. Psychosis: impact on the patient and family. Neurology 1999;52(supp13):S1416.

7 Fahn S. Parkinsonism. In: Rowland LP, eds. Merritt's textbook of neurology. Baltimore, MD: Williams and Wilkins, 1995.

8 Moskovitz C, Moses H, Klawans HL. Levodopa-induced psychosis: a kindling phenomenon. Am $\mathcal{f}$ Psychiatry 1978;135:669-75.

9 Nausieda P, Glantz R, Weber S, et al. Psychiatric complications of levodopa therapy in Parkinson's disease. Adv Neurol 1984;40:271-7.

10 Aarsland D, Larson JP, Cummings JL, et al. Prevalence and clinical correlates of psychiatric symptoms in Parkinson's disease: a community-based study. Arch Neurol 1999, 56:595-601.

11 Tanner CM, Vogel C, Goetz CG, et al. Hallucinations in Parkinson's disease: a population study. Ann Neurol 1983;14:136.

12 Sanchez-Ramos JR, Ortoll R, Paulson GW. Visual hallucinations associated with Parkinson's disease. Arch Neurol 1996;53:1265-8.

13 Commella CL, Tanner CM, Ristanovic RK. Polysomnographic sleep measures in Parkinson's disease patients with treatment induced hallucinations. Ann Neurol 1993:34: 710-14.

14 Meco G, Bonifati MG, Cusimano GI, et al. Hallucinations in Parkinson's disease: neuropsychological study. Ital $\mathcal{f}$ Neurol Sci 1990;11:373-9.

15 Pahwa R, Lyons K, Hubble J, et al. Hallucinations in Parkinson's disease patients correlate with cognitive impairment [abstract]. Mov Disord 1995;10:695.

16 Masterman DL, Cummings JL. Parkinson's disease update. Part II: neuropsychiatric aspects. Clin Geriatr Med 1998;6: $76-81$.

17 Holroyd S, Keller AS. A study of visual hallucinations in Alzheimer's disease. Am F Geriatr Psychiatry 1995;3:198205.

18 Holroyd S, Rabins PV, Finkelstein D, et al. Visual hallucinations in patients from an ophthalmology clinic and medical clinic population. F Nerv Ment Dis 1994;183:273-6.

19 Holroyd S, Rabins PV, Finkelstein D, et al. Visual hallucinations in patients with macular degeneration. Am f Psychiatry 1992;149:1701-6.

20 Diederich NJ, Goetz CG, Raman R, et al. Primary deficits in visual discrimination is a risk factor for visual hallucinations in Parkinson's disease Neurology 1997;48:A181.

21 Ward CD, Gibb WR. Research diagnosis criteria for Parkinson's disease. Adv Neurol 1990:53:245-9.

22 Hughes AJ, Daniel SE, Kilford L, et al. The accuracy of clinical diagnosis of idiopathic Parkinson's disease: a clinical diagnosis of idiopathic Parkinson's disease: a 1992;55:181-4.

23 Hughes AJ, Ben-Shlomo Y, Daniel SE, et al. What features improve the accuracy of clinical diagnosis in Parkinson's disease: a clinicopathologic study. Neurology 1992;42: $1142-6$

24 American Psychiatric Association. Diagnostic and statistical manual of mental disorders. 4th ed. Washington, DC: American Psychiatric Association, 1994.

25 Brandt J, Spencer M, Folstein M. The telephone interview for cognitive status. Neuropsychiatry Neuropsychol Behav Neurol 1988;1:111-17.

26 Folstein MF, Folstein SE, McHugh PR. Mini-mental state: a practical method for grading the cognitive state of patients for the clinicion fPsychiatr Res 1975;12:189-98.

patients for the clinician. F Psychiatr Res 1975;12:189-98.
27 Brink TL, Rose TL, Lum O, et al. Development and validation of a geriatric depression screening scale: a preliminary report. F Psychiatr Res 1983 17:37-49.

28 Norris JT, Gallagher D, Wilson A, et al. Assessment of depression in geriatric medical outpatients: the validity of two screening measures. f Am Geriatr Soc 1987;35:989-95.

29 McGivney SA, Mulvihil M, Taylor B. Validating the GDS depression screen in the nursing home. $f \mathrm{Am}$ Geriatr Soc 1991;42:490-2.

30 Koenig HG, Meader KG, Cohen HJ, et al. Self rated depression scales and screening for major depression in the older hospitalized patient with medical illness. $7 \mathrm{Am}$ Geriatr older hospitalized patient

31 Fahn S, Elton RC, and members of the UPDRS Development Committee. Unified Parkinson's disease rating scale. In: Fahn S, Marsden DC, Goldstein M, et al, eds. Recent developments in Parkinson's disease. Volume II Florheim Park, New Jersey: Macmillan, 1997:153-63.

32 Pappert EJ, Goetz CG, Ramon R, et al. Stability of hallucinations and illusions in Parkinson's disease: 18 month prospective study. Mov Disord 1998;13(suppl):90.

33 Goetz CG, Pappert EJ. Blasocci LM, et al. Intravenous levodopa in hallucinating Parkinson's disease patients: high dose challenge does not precipitate hallucinations. Neuroldose challenge does

34 Bodis-Wollner I, Yahr MD. Measurement of visual evoked potentials in Parkinson's disease. Brain 1978;101:661-71. 35 Bodis-Wollner I, Tagliati M. The visual system in Parkinson's disease. Adv Neurol 1993;60:390-4. 